\title{
LA RESPONSABILIDAD SOCIAL EMPRESARIAL EN URUGUAY: UNA VISIÓN COMPARADA DESDE LA CIUDADANIÍA Y DESDE LAS EMPRESAS*
}

\author{
Zuleika Ferre**; Natalia Melgar***

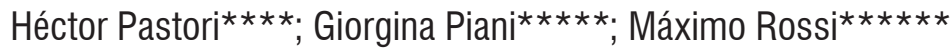 \\ Recibido: agosto 01 de 2010 \\ Aceptado: septiembre 28 de 2011
}

\section{RESUMEN}

El objetivo de este trabajo es describir el comportamiento de las empresas uruguayas y la opinión de los ciudadanos sobre la responsabilidad social empresarial (RSE). Para ello se utilizaron datos de las encuestas aplicadas a la opinión pública y las empresas sobre RSE. El trabajo presenta un análisis descriptivo comparado de los principales resultados de dichas encuestas con el objetivo de aportar al conocimiento de un tema en el que existen pocos antecedentes de investigación académica en Uruguay. El análisis de los datos muestra un cierto grado de conocimiento de la población sobre el tema de RSE. Se destaca que los ciudadanos tienen una opinión más favorable en cuanto al desempeño de las empresas que el de las propias empresas. Además, existe un cierto balance entre las acciones empresariales en RSE y la valoración de la opinión pública de aquellas áreas consideradas importantes.

\section{PALABRAS CLAVE}

Responsabilidad social, opinión pública, empresas, Uruguay.

\section{CLASIFICACIÓN JEL} M14, D10, D22, L14

\section{CONTENIDO}

Introducción; 1. Antecedentes: evolución del concepto de RSE; 3. Los datos; 4. Principales resultados; 5 . Conclusiones; Bibliografía.

\footnotetext{
Este artículo es producto del proyecto de investigación "Responsabilidad Social en Uruguay" desarrollado por un grupo de investigadores adscritos al del Departamento de Economía, Facultad de Ciencias Sociales, Universidad de la República (UdelaR), el cual se realizó entre 2006 y 2007. El proyecto fue financiado por la Comisión Sectorial de Investigación Científica de la Universidad de la República y la Asociación Cristiana de Dirigentes de Empresa.

* Licenciada en Sociología y magíster en Demografía, Facultad de Ciencias Sociales, UdelaR, Montevideo, Uruguay. Profesora adjunta del Departamento de Economía, Facultad de Ciencias Sociales, UdelaR, Montevideo, Uruguay. Correo electrónico: zuleika@decon. edu.uy.

**: Licenciada en Economía, Facultad de Ciencias Sociales, UdelaR, Montevideo, Uruguay; magíster en Economía, Facultad de Ciencias Sociales, UdelaR; PhD in Economics, Universidad de Granada, Granada, España. Asistente de investigación, de Economía, Facultad de Ciencias Sociales, UdelaR, Montevideo, Uruguay. Correo electrónico: nmelgar@decon.edu.uy.

*.** Ingeniero Agrónomo, Facultad de Agronomía, UdelaR; MBA, Universidad ORT, Montevideo, Uruguay. Profesor Adjunto, Departamento de Economía, Facultad de Ciencias Sociales, UdelaR, Montevideo, Uruguay. Correo electrónico: hector@decon.edu.uy.

***** Licenciada en Sociología, Facultad de Ciencias Sociales, UdelaR; magíster en Diseño de encuestas, Universidad de Michigan, Estados Unidos. Asistente de investigación, Departamento de Economía, Facultad de Ciencias Sociales, UdelaR, Montevideo, Uruguay. Correo electrónico: giorgina@decon.edu.uy.

****** Economista, Facultad de Ciencias Económicas, UdelaR; doctor en Economía, Universidad de Granada, España. Profesor e investigador, Departamento de Economía, Facultad de Ciencias Sociales, UdelaR, Montevideo, Uruguay. Correo electrónico: mito@ decon.edu.uy.
} 


\section{CORPORATE SOCIAL RESPONSIBILITY IN URUGAY: A COMPARATIVE APPROACH FORM THE CITIZENSHIP AND FROM THE COMPANIES}

\section{ABSTRACT}

The objective of this paper is to describe the behavior of Uruguayan companies and the opinion that Uruguayan citizens have about corporate social responsibility (CSR). For this purpose, data was collected form surveys answered by citizens over CSR. This paper presents a comparative descriptive analysis on the main results obtained by these surveys with the goal of contributing to a topic that has little academic research background in Uruguay. The data analysis shows a certain degree of acknowledgement by the citizens over this specific topic (CSR). The analysis also shows that citizens have a better perception of companies than companies themselves. In addition, there is an existent balance between the actions taken by the company in regards to CSR and the public opinion in those areas that are considered important.

KEY WORDS

Social responsibility, Uruguay, Public opinion, Companies

JEL CLASSIFICATION

M14, D10, D22, L14

\section{CONTENT}

Introduction; 1. Background; Evolution of the CSR concept ; 3. Data; 4. Main results;

5. Conclusions; Bibliography.

\section{A RESPONSABILIDADE SOCIAL EMPRESARIAL NO URUGUAI: UMA VISÃO COMPARADA DESDE A CIDADANIA E DESDE AS EMPRESAS.}

\section{RESUMO}

O objetivo deste trabalho é descrever o comportamento das empresas uruguaias e a opinião dos cidadãos sobre a responsabilidade social empresarial (RSE). Usaram-se dados de sondagens feitos aos cidadãos e as empresas sobre RSE. O artigo apresenta uma análise comparativa descritiva dos principais resultados destas pesquisas com o objetivo de contribuir para o conhecimento dum assunto sobre o qual há pouca pesquisa acadêmica de fundo no Uruguai. A análise dos dados mostra um certo grau de conhecimento da população sobre a questão da RSE. Ressalta-se que os cidadãos tem uma opinião mais favorável em relação ao desempenho das empresas que as próprias empresas. Além disso, há um certo equilíbrio entre RSE e ações corporativas na avaliação da opinião pública nas áreas consideradas importantes.

\section{PALAVRAS-CHAVE}

Responsabilidade social, Uruguai, opinião pública, Empresas

\section{CLASSIFICAÇÃO JEL} M14, D10, D22, L14

\section{CONTEÚDO}

Introdução; 1. Antecedentes: Evolução do conceito de RSE, 3. Os dados; 4. Principais resultados; 5 . Conclusões; Bibliografia. 


\section{INTRODUCCIÓN}

En el transcurso de los últimos cuarenta años (1970-2010) la concepción de que los problemas sociales debían ser resueltos casi exclusivamente desde el ámbito público ha cambiado, incrementándose notoriamente el papel de las organizaciones no gubernamentales. En este proceso de sustitución de las acciones que correspondían al ámbito fundamentalmente público, ingresan, como nuevos actores las empresas, tanto públicas como privadas, a desarrollar actividades de Responsabilidad Social Empresarial (RSE).

El interés por la RSE es creciente en el ámbito internacional y en especial en los países en vía de desarrollo. En este sentido las acciones de las empresas encuadradas en el concepto de RSE son visualizadas como:

- Un medio para fortalecer las políticas económicas que permitan el desarrollo sustentable

- Mecanismos de fortalecimiento de las estrategias de competitividad

- Posicionamiento de las exportaciones en el mercado internacional

- Una forma de atraer inversores extranjeros

- Un nuevo enfoque para luchar contra la pobreza

En Uruguay se destacan las acciones de organizaciones empresariales que buscan fomentar la RSE en sus empresas. La Asociación Cristiana de Dirigentes de Empresa (ACDE) desde el año 2003 promociona la RSE en Uruguay con la elaboración un Índice de Responsabilidad Social Empresarial (IRSE), que evalúa la evolución del comportamiento de las empresas a lo largo del tiempo. Por otra parte, la organización Desarrollo de la Responsabilidad Social (DERES) nuclea desde el año 2001 a un conjunto de empresas e instituciones que trabajan en la temática de la responsabilidad social, lo que le ha permitido crear herramientas, instrumentos y materiales de apoyo que faciliten su implementación en Uruguay.

El presente trabajo tiene como principal objetivo describir el comportamiento de las empresas uruguayas y la opinión de los ciudadanos en relación a RSE. Para ello se utilizaron datos de las encuestas sobre RSE realizada a la Opinión Pública y Empresas las cuales fueron aplicadas por el convenio dECONACDE $E^{1}$ en los años 2006 y 2007, respectivamente (Ferre y otros, 2007 y Piani y otros, 2007).

Del análisis se destaca que más de la mitad de la población uruguaya dice haber escuchado hablar de RSE. Por otra parte, se encuentra que la ciudadanía le asigna un importante rol al gobierno y a las empresas en apoyar proyectos comunitarios y de caridad. Por último, se destaca la existencia de una cierta concordancia entre las acciones de RSE implementadas por las empresas y la valoración que la opinión pública realiza de las áreas consideradas como más importantes. El ámbito que involucra a los trabajadores y empleados (capacitación, promoción de participación, promoción de la salud, mejoras en la seguridad, etc.) aparece como el área de mayor importancia.

Este artículo se organiza de la siguiente forma. En la sección 2 se presentan los antecedentes que surgen de la revisión bibliográfica. En la sección 3 se describe la metodología de las dos encuestas utilizadas. En la sección 4 se analizan los resultados. Finalmente, en la sección 5 se presentan las conclusiones.

\section{ANTECEDENTES}

El análisis de los antecedentes teóricos muestra que no existe una única definición de la noción de RSE. A pesar de esto, existe cada vez mayor consenso sobre los límites del concepto y la definición de una serie de elementos compartidos que hacen parte de la noción de RSE.

McWilliams y Siegel (2001) entienden la RSE como el conjunto de acciones que promueven algún bien social más allá de los intereses de las empresas y de las exigencias de la ley. En este marco, la RSE

DECON: Departamento de Economía (Facultad de Ciencias Sociales, Universidad de la República) y ACDE: Asociación Cristiana de Dirigentes de Empresa. 
se define como el compromiso de las empresas a contribuir con el desarrollo económico de los empleados, sus familias, la comunidad y la sociedad en su conjunto con el objetivo de mejorar la calidad de vida de todos los grupos involucrados. Por lo tanto, la RSE puede ser vista como el mecanismo mediante el cual las empresas u organizaciones contribuyen a la estabilidad y mejora de la comunidad.

Carroll (1998) propone cuatro dimensiones que caracterizan la responsabilidad que las empresas deben asumir ante la sociedad. En primer lugar, responsabilidad económica, que se deriva del cumplimiento de su función tradicional, o sea de la producción de bienes y servicios con el objetivo de maximizar el beneficio. En segundo lugar, responsabilidad legal, esto es el respeto a las leyes que regulan su actividad. En tercer lugar, responsabilidad ética, que refiere al conjunto de actividades que son vistas como correctas o incorrectas por la sociedad, aunque no se encuentren enmarcadas en el ámbito legal. Y por último, responsabilidad social, que responde al conjunto de actividades que no son una exigencia de la sociedad pero que son consideradas satisfactorias, como por ejemplo: apoyo financiero a obras sociales, actividades de promoción de la comunidad en la que la empresa se inserta o el apoyo de eventos relevantes para la sociedad.

Si bien es difícil ubicar con precisión el origen del concepto, diversos autores coinciden en que es a partir de los años cincuenta que la noción de RSE comienza a circular entre las empresas estadounidenses con la publicación de Bowen (1953), "Social Responsibilities of the Businessman". Según Aparicio y Valdés (2009), con esta publicación toma fuerza la noción de las obligaciones que los hombres de negocios tienen en la ejecución de acciones o políticas y su correspondencia con los valores de la sociedad.

Desde ese momento si bien la difusión y consolidación de las prácticas de responsabilidad social fue creciente en el ámbito mundial, es recién en los años noventa cuando comienzan a tomar forma el concepto y las acciones que definen la RSE. Producto de este proceso es el surgimiento de códigos éticos y de conducta que delinean la noción de lo que hoy en día se conoce como RSE. Ejemplo de esto son los diez principios contenidos en United Nations Global Compact (1999), los cuales deben ser implementados por los miembros y que refieren a cuatro categorías relacionadas con los derechos humanos, estándares laborales, medioambiente y corrupción. Por otra parte, la Commission European Communities (2001) establece estrategias para promover las contribuciones de las empresas al progreso social y del medioambiente. A su vez las normas SA-8000 y el estándar AA-1000 (Institute of Social Ethical Accountability) establecen códigos para lograr estandarizar el manejo ético de los negocios. Por último, con la creación de la norma ISO 26000 en Uruguay (IUNT, 2010), se genera una nueva guía para las acciones de la responsabilidad social en las organizaciones.

En este contexto, diversos autores (Porter y Kramer, 2006; De la Cuesta, 2004; Aparicio y Valdés, 2009) se preguntan cuáles son las motivaciones para que se difundan las políticas de RSE. Estas motivaciones o elementos tienen que ver con intereses morales, sociales o económicos y, si bien es posible separarlos, deben ser analizados en forma conjunta, ya que lo común es que en la práctica interactúen unos con otros.

En este sentido, las justificaciones de tipo moral no analizan el beneficio económico ligado a la gestión socialmente responsable. Las empresas son entes con responsabilidad no solo económica, sino que cumplen, además, con un papel social en la comunidad; por tanto deben tener comportamientos acordes a ese rol. La segunda línea argumental se refiere a un enfoque de mejora en los negocios a partir de los vínculos existentes o potenciales entre la calidad de las relaciones de la empresa con los grupos o individuos involucrados o que de alguna manera se ven afectados por la actividad de la misma. Estos grupos son definidos por la literatura 
como stakeholders y refieren a empleados, inversores, proveedores, consumidores y la comunidad.

Los activistas sociales y ambientales, muchas veces agrupados en organizaciones no gubernamentales -locales o globales- generan presión en las empresas con el objetivo de compensar las debilidades institucionales y alinear el comportamiento organizacional con el objetivo social y medioambiental de desarrollo sustentable. Es esta presión la que induce a las empresas a mostrar su responsabilidad social y minimizar el desgaste con los stakeholders (Becchetti, Di Giacomo y Pinnachio, 2008). En este contexto, la RSE se originaría no por decisiones autónomas de los directores, sino por presiones externas de los consumidores o instituciones.

En general, se señala en la literatura que la RSE es una forma de autorregulación, es decir, va más allá de la regulación formal establecida por el Estado. Por ejemplo, las externalidades negativas que son generadas por la actividad productiva de las empresas tradicionalmente son resueltas a través de la regulación formal vía la imposición de cuotas, impuestos, subsidios, entre otras medidas. Con respecto a esta problemática, las acciones que constituyen RSE serían políticas privadas que complementarían estas políticas públicas, es decir, una empresa que simplemente cumple con la regulación impuesta por el Estado no realiza acciones de RSE.

Por otro lado, el conjunto de prácticas que podría realizar una empresa con el objetivo de ser socialmente responsable, si bien constituyen una herramienta que mejora las relaciones con el medio, son acciones que contribuyen a generar valor, ya sea mediante la optimización de procesos, reducción de costos o que potencialmente podrían provocar diferenciación de productos. En cualquiera de estos casos, la empresa debería invertir en esas acciones independientemente de su RSE (Porter y Kramer, 2006).

Además de la conciencia empresarial, la RSE es demandada por los consumidores cada vez más atentos al comportamiento socialmente responsable de las empresas. Esta exigencia, que ha crecido en importancia, de los miembros de la comunidad sobre el comportamiento de las empresas en el entorno social constituye un significativo incentivo para que las empresas vayan más allá de los requerimientos regulatorios, y permite incorporar en su acción aspectos que constituyen la responsabilidad social. La evidencia indica que existen consumidores dispuestos a pagar precios más altos por productos de empresas socialmente responsables o que penalizan a las empresas que no siguen dichos comportamientos (Fleishman-Hillard-NCL, 2005).

La literatura sobre el tema muestra que los ciudadanos conscientes constituyen un agente que internaliza las externalidades generadas a través de su consumo cuando decide qué producto comprar, a pesar de que el impacto de su comportamiento individual es insignificante en el problema en cuestión (contaminación ambiental, respeto a los derechos individuales, etc.). La existencia de estos ciudadanos conscientes realimenta las estrategias y políticas que adoptan las empresas con respecto a actividades socialmente responsables. En el caso extremo de ausencia de regulación, la existencia de ciudadanos conscientes constituirá un incentivo para la adopción de prácticas socialmente responsables por parte de las empresas, dado que este atributo formará parte de las decisiones de consumo de los individuos.

Autores como Zadek (2004) proponen analizar la RSE desde dos dimensiones analíticas: el aprendizaje organizacional de las empresas hacia la RSE y la madurez de las empresas respecto al asunto social en cuestión. En lo que respecta a la primera dimensión, las empresas recorren un camino de aprendizaje de cinco etapas hacia la RSE. Estas etapas y sus características son:

1. Defensiva, negación de efectos y responsabilidades por su accionar.

2. Obediencia, se establece una política de obediencia que implica asumir los cos- 
tos como parte del que hacer empresarial.

3. Directivo, se da responsabilidad a los gerentes sobre los asuntos sociales y su resolución, se integran prácticas socialmente responsables a las actividades del día a día.

4. Estratégico, se integran los asuntos sociales a las estrategias centrales de la empresa.

5. Civil, se promueve una amplia participación del sector empresarial en RSE.

La segunda dimensión de análisis, que refiere a la madurez con respecto al asunto social, es definida en cuatro etapas:

1. Latente, solo las comunidades de activistas y algunas ONG tienen conciencia del tema, existe débil evidencia científica y, en consecuencia, el asunto es ampliamente ignorado o poco valorado por la comunidad de negocios.

2. Emergente, existe cierto interés en la comunidad política y en los medios de comunicación; comienza a desarrollarse investigación sobre el tema aunque las evidencias aún son débiles; las empresas líderes experimentan aproximaciones para tratar este asunto.

3. Consolidado, emerge un cuerpo de prácticas de negocio alrededor del asunto social, se establecen iniciativas voluntarias, hay litigios en curso y se percibe la necesidad de legislación, se crean estándares voluntarios y se llevan adelante acciones colectivas.

4. Institucionalizado, se legisla y se establecen normas comerciales, las actividades involucradas se convierten en una parte normal del modelo de excelencia de gestión.

Es claro que existe relación entre el avance que realizan las empresas y en el avance social de los distintos temas. Las empresas para mantenerse en una zona de oportunidades deberían ir por delante del desarrollo social.

En resumen, la expansión de las prácticas socialmente responsables ha sido acompasada por el desarrollo de un marco conceptual que le ha dado los fundamentos teóricos en los que se sustenta la
RSE y el marco de acción para su implementación.

En Uruguay, tal como se señaló anteriormente, las actividades de RSE han tenido un importante impulso en los últimos diez años (2000-2009), fundamentalmente promovido por la acción de organizaciones de empresas tendentes a nuclear y fomentar las prácticas socialmente responsables. A pesar de esto, prácticamente no existen estudios empíricos basados en muestreos estadísticamente representativos que permitan describir y conocer la realidad nacional sobre RSE.

Las encuestas que se presentan en la sección siguiente tuvieron como objetivo cubrir ese vacío de información y se enmarcaron en un proyecto más amplio sobre RSE en Uruguay, realizado entre el Departamento de Economía de la Facultad de Ciencias Sociales de la Universidad de la República y la ACDE (Ferre y otros, 2007; Piani y otros, 2007).

El presente trabajo pretende describir, a partir de datos objetivos y confiables, la situación de la RSE en Uruguay abordada desde la perspectiva de dos actores que juegan diferentes roles en el desarrollo de las prácticas de RSE: las empresas y los ciudadanos.

\section{DATOS}

\subsection{Encuesta de Opinión Pública (EOP) sobre RSE}

La EOP estuvo dirigida a la población mayor de 17 años residentes en viviendas particulares de Montevideo e Interior Urbano (localidades urbanas con población igual o mayor a 5.000 habitantes). La aplicación de las encuestas se realizó entre los meses de noviembre y diciembre de 2006.

Para la selección de la muestra se utilizó el marco muestral del Censo 2001 Fase I, elaborado Instituto de Nacional de Estadística (INE). El diseño muestral fue probabilístico, estratificado y por conglomerados donde la unidad última de selección fue una persona mayor de 17 años seleccionada al azar entre todos los miembros del hogar que cumplieron 
con esa condición. El tamaño muestral fue de 1.031 viviendas. La tasa de respuesta a la EOP fue de $70,6 \%$, la cual se encuentra dentro de los estándares internacionales para este tipo de encuestas. (Piani y otros, 2007)

La finalidad principal de la encuesta de EOP fue conocer el nivel de información y conocimiento de la población respecto a la RSE, así como las imágenes, actitudes y opiniones sobre el tema, presentes en los encuestados al momento de contestar. El diseño del cuestionario se realizó a partir de las experiencias de encuestas similares en Estados Unidos y Latinoamérica (NCL, 2005; PNUD _ ProHumana, 2002), las cuales fueron ajustadas a la realidad uruguaya y a los recursos financieros del proyecto.

Cuadro 1. Las principales características de los encuestados (en \%)

\begin{tabular}{|l|l|l|}
\hline \multirow{1}{*}{ Característica } & \multicolumn{1}{|c|}{ Categorías } & Frecuencia \\
\hline \multirow{4}{*}{ Sexo } & Hombres & 46 \\
\cline { 2 - 3 } & Mujeres & 54 \\
\hline \multirow{4}{*}{ Tramosión de edad } & Montevideo & 57 \\
\cline { 2 - 3 } & Resto del país urbano & 43 \\
\cline { 2 - 3 } & 35 a 49 años & 34 \\
\cline { 2 - 3 } & 50 a 69 años & 29 \\
\cline { 2 - 3 } & 70 y más & 26 \\
\hline \multirow{4}{*}{ Nivel educativo } & Primaria & 11 \\
\cline { 2 - 3 } & Secundaria & 36 \\
\cline { 2 - 3 } & Terciaria & 46 \\
\hline \multirow{3}{*}{$\begin{array}{l}\text { Condición de } \\
\text { actividad }\end{array}$} & Inactivo & 18 \\
\cline { 2 - 3 } & Desocupado & 12 \\
\cline { 2 - 3 } & Empleado & 56 \\
\hline
\end{tabular}

Fuente: Elaboración propia a partir de la EOP

Se debe tener en cuenta que debido a que la RSE no resulta un tema habitual de reflexión y conversación entre la población uruguaya, los encuestados se encontraron en su mayoría, ante la necesidad de elaborar sus opiniones, imágenes y actitudes al momento de contestar la encuesta. Los entrevistados debieron hacer uso de la infor- mación acumulada que pudo ser recordada en ese momento, así como de opiniones y actitudes ya formadas sobre temas que el encuestado relacionó a la RSE. Por tal motivo, tanto en la elaboración del cuestionario como en su aplicación, se tomó especial cuidado en facilitar la comprensión de las preguntas a través de misivas cortas, materiales visuales (tarjetas), lectura pausada de las preguntas y repetición de las mismas. En el cuadro 1 se presentan las principales características socioeconómicas de la muestra.

\subsection{Encuesta a empresas (EE) sobre RSE}

La aplicación de las encuestas a las empresas se realizó entre los meses de abril y junio de 2007. La muestra es representativa de empresas de 50 o más personas ocupadas en los sectores comercio, industria y servicios. El diseño muestral fue estratificado simple a partir de los datos del Registro de Actividad Económica Permanente de 2005 (INE). Se entrevistaron 95 empresas de Montevideo y Zona metropolitana.

La EE se realizó a través de una modalidad mixta de contacto con la empresa, vía teléfono y mail, y una posterior visita del encuestador en donde se aplicó el cuestionario en forma de entrevista personal. El diseño del formulario de la EE tuvo como antecedente directo el Índice de Responsabilidad Social Empresarial elaborado por ACDE (2005 y 2006). Se realizaron preguntas tendentes a recoger información sobre programas, actividades o planes de las empresas acerca de las cuatro dimensiones de la RSE: "empleados", "accionistas", "clientes y competidores", y "comunidad, Estado y medioambiente". Con el objetivo de evitar la tendencia natural al sobre reporte de actividades de RSE, se solicitó a los encuestados que brindaran ejemplos de cada una de aquellas actividades declaradas, tomándose como período de referencia las actividades realizadas en el año 2006. Se incluyeron, además, preguntas que recogieran información sobre la imagen y el rol de las empresas en la RSE, así como una serie de preguntas de 
caracterización de la empresa (Ferre y otros, 2007).

A los efectos de tener una primera lectura de los datos obtenidos, se eligieron cuatro variables de clasificación que generalmente explican las diferencias en los comportamientos de las empresas, las cuales se presentan en el cuadro 2.

Cuadro 2. Las principales características de las empresas encuestadas (en \%)

\begin{tabular}{|c|c|c|}
\hline Característica & Grupos & $\%$ \\
\hline \multirow{3}{*}{ Sector } & Comercio & 16,0 \\
\hline & Industria & 30,0 \\
\hline & Servicios & 54,0 \\
\hline \multirow{5}{*}{ Tamaño } & Entre 50 y 100 empleados & 43,2 \\
\hline & Entre 101 y 150 empleados & 15,2 \\
\hline & Entre 151 y 200 empleados & 14,9 \\
\hline & Entre 201 y 300 empleados & 9,1 \\
\hline & Más de 300 empleados & 17,6 \\
\hline \multirow{3}{*}{$\begin{array}{l}\text { Propiedad } \\
\text { del capital }{ }^{1}\end{array}$} & Capitales nacionales & 65,8 \\
\hline & Capitales extranjeros & 10,0 \\
\hline & $\begin{array}{l}\text { Capital nacional con acuerdo } \\
\text { con extranjeros }\end{array}$ & 24,2 \\
\hline \multirow{2}{*}{$\begin{array}{l}\text { Antigüedad } \\
\text { de la } \\
\text { empresa }^{2}\end{array}$} & Inicio actividades hasta 1982 & 67,0 \\
\hline & $\begin{array}{l}\text { Inicio actividades a partir de } \\
1983\end{array}$ & 33,0 \\
\hline \multicolumn{3}{|c|}{$\begin{array}{l}\text { Notas: } \\
\text { 1) Se consideró capitales extranjeros a aquellas empresas con } \\
\text { participación de capitales extranjeros o que son filiales de } \\
\text { multinacional. Las empresas de capital nacional con acuerdo } \\
\text { con extranjeros son las que tienen acuerdos tales como; fran- } \\
\text { quicias, licencias, marcas y patentes, acuerdos asociativos para } \\
\text { producción y/o comercialización, etc. } \\
\text { 2) Se consideró como año de corte para el inicio de actividades, } \\
1982 \text { ya que las empresas creadas posteriormente a esa fecha } \\
\text { deberían estar marcadas por la apertura comercial lo que las } \\
\text { diferenciaría de las empresas tradicionales. }\end{array}$} \\
\hline
\end{tabular}

Fuente: Elaboración propia a partir de la EE.

\section{PRINCIPALES RESULTADOS}

A continuación se presentan los principales resultados de ambas encuestas. Desde un enfoque comparativo, se pretende contrastar la opinión que la población tiene sobre la RSE con lo que declaran las empresas en esta materia.

\subsection{Conocimiento y nivel de información}

Gráfico 1. ¿Escuchó hablar alguna vez de RSE? (\%)

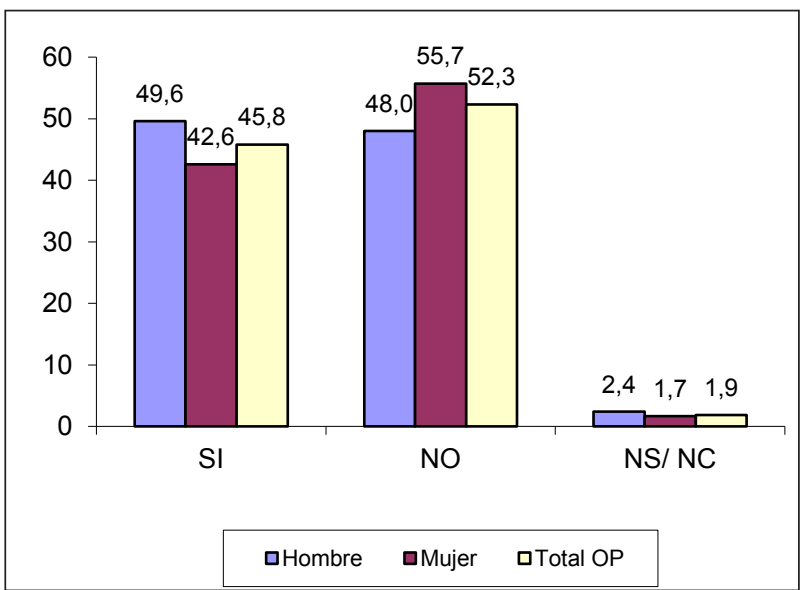

Fuente: Elaboración propia a partir de la encuesta EOP

Un primer objetivo de la EOP fue relevar el nivel de información de la población sobre RSE. En este sentido se le preguntó a los encuestados si alguna vez escucharon hablar de RSE, donde casi la mitad de la población declara haber escuchado del tema. Al distribuir las respuestas por el sexo, los hombres tienen mayor nivel de conocimiento del tema que las mujeres.

Cuadro 3. Conocimiento del tema por edad, educación y actividad (\%).

\begin{tabular}{|l|l|c|}
\hline \multirow{4}{*}{ Característica } & \multicolumn{1}{|c|}{ Grupos } & $\begin{array}{c}\text { ¿Escuchó hablar sobre RSE? } \\
\text { (\% de respuestas afirmativas) }\end{array}$ \\
\hline \multirow{4}{*}{ Edad } & 18 a 34 años & 39,1 \\
\cline { 2 - 3 } & 35 a 49 años & 49,1 \\
\cline { 2 - 3 } & 50 a 69 años & 52,3 \\
\cline { 2 - 3 } & Más de 70 años & 42,6 \\
\hline \multirow{4}{*}{ Educación } & Primaria & 32,5 \\
\cline { 2 - 3 } & Secundaria & 47,0 \\
\cline { 2 - 3 } & Terciaria & 69,1 \\
\hline \multirow{4}{*}{ Actividad } & Inactivo & 40,4 \\
\cline { 2 - 3 } & Desempleado & 37,6 \\
\cline { 2 - 3 } & Ocupados & 50,6 \\
\hline
\end{tabular}

Fuente: Elaboración propia a partir de la encuesta EOP 
Como se esperaba, dado que el nivel de información de los ciudadanos está relacionado con el nivel de educación, a mayor nivel educativo aumenta el porcentaje de personas que responden afirmativamente a la pregunta "¿Alguna vez escuchó hablar de RSE?". Asimismo, se encuentra que el nivel de conocimiento sobre el tema aumenta a medida que la edad es mayor, excepto para los adultos mayores en donde se registran porcentajes similares a los de la población más joven. Por último, se encuentra que los ocupados presentan mayores porcentajes de respuestas afirmativas respecto a los desempleados e inactivos.

Gráfico 2. Conocimiento del tema por región (\%)

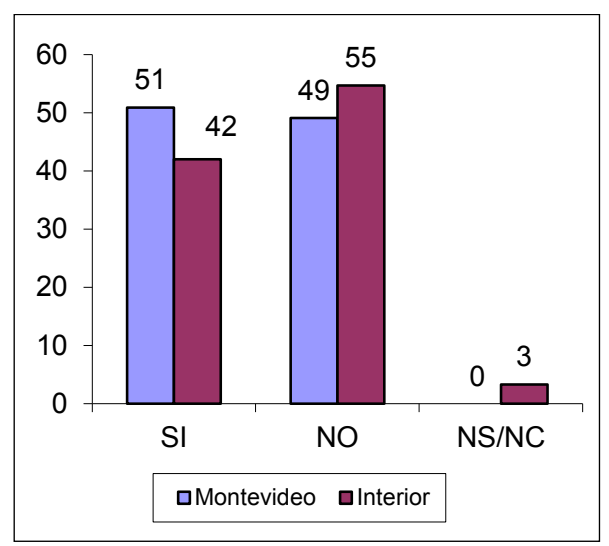

Fuente: Elaboración propia a partir de la encuesta EOP

Se destacan diferencias por región. El conocimiento que los ciudadanos de Montevideo tienen sobre el tema es mayor que el del resto del país urbano. De hecho, mientras que en el interior urbano 3,3\% de los encuestados dice no saber o no querer contestar, en Montevideo este porcentaje es nulo. En general, la opción "no sabe/no contesta" constituye una opción para los encuestados que no conocen o no saben sobre un tema y se sienten incómodos de admitirlo. Esto podría explicar parte de la diferencia observada entre las dos regiones.

Debido a que no existen en Uruguay antecedentes empíricos sobre qué atributos le otorgan al concepto de RSE la población en general y las empresas, se decidió incorporar en ambas encuestas una pregunta abierta (sin opciones de respuestas) con el objetivo de captar la gran diversidad de ideas vinculadas al tema. Para su procesamiento se agruparon las respuestas a partir de la presencia y referencias a conceptos e ideas similares ${ }^{2}$.

Gráfico 3. ¿Qué entiende por RSE?

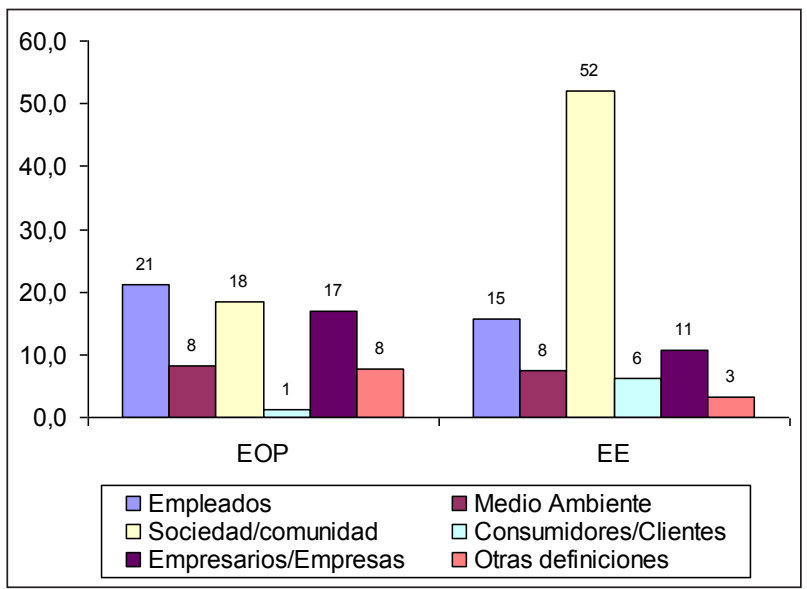

Nota: Los valores no suma 100 porque faltan los No sabe/No contesta.

Fuente: Elaboración propia a partir de la EE y EOP

Entre los encuestados de la población que aportaron su definición de RSE, los conceptos mayoritariamente incluidos en la definición fueron aquellos vinculados a los trabajadores, la sociedad y las empresas. Se formuló la misma pregunta a las empresas, donde más de la mitad asocia RSE a elementos vinculados a la acción en pro de la comunidad y la sociedad en general.

\subsection{Evaluación de la RSE}

Al comparar la calificación que realiza la población general y las empresas respecto al desempeño de las empresas en temas de RSE, se observa que

\footnotetext{
2 Luego de obtener los elementos conceptuales espontáneos relacionados con el término RSE, el cuestionario aportaba una definición estándar de RSE a fin de proporcionar un mismo marco conceptual para responder el resto del cuestionario. A fin de facilitar la comprensión de esta definición el encuestador entregaba una tarjeta al encuestado para que este pudiera seguir la lectura. "Por RSE se entiende el comportamiento responsable y comprometido de la empresa con respecto a los empleados, clientes, proveedores, accionistas, la comunidad y el medio ambiente donde esta produce $y /$ o vende sus productos o servicios".
} 
en su autoevaluación las empresas, en promedio, son más críticas, agrupándose las respuestas entre las notas 2 y 3 . En cambio, la opinión pública presenta mayor frecuencia en el extremo positivo de la escala. En paralelo a esto, se observa también una evaluación positiva cuando comparan el desempeño de las empresas respecto a dos años atrás, casi la mitad de los encuestados manifiesta que la RSE actual es mucho o algo mejor.

Gráfico 4. Calificación del desempeño de las empresas en RSE (\%)

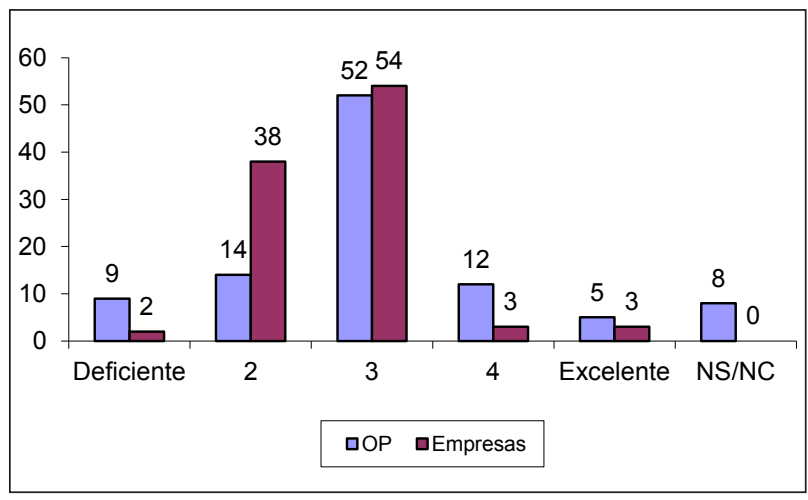

Fuente: Elaboración propia a partir de la EOP y EE.

Cuadro 4. Calificación del desempeño de las empresas en RSE dos años atrás (\%)

\begin{tabular}{|l|c|}
\hline \multicolumn{1}{|c|}{ Calificación } & $\%$ \\
\hline Mucho peor & 1.3 \\
\hline Algo peor & 7.0 \\
\hline Más o menos igual & 38.8 \\
\hline Algo mejor & 40.1 \\
\hline Mucho mejor & 7.2 \\
\hline No sabe/No contesta & 5.6 \\
\hline Total & 100 \\
\hline
\end{tabular}

Fuente: Elaboración propia a partir de la EE.

$\mathrm{Al}$ analizar las respuestas de las empresas por sector de actividad al que pertenecen, la industria se muestra como el sector más crítico en cuanto al desempeño de las empresas uruguayas en RSE, seguido del sector comercio.
Gráfico 5. Calificación del desempeño de las empresas en RSE por sector (\%)

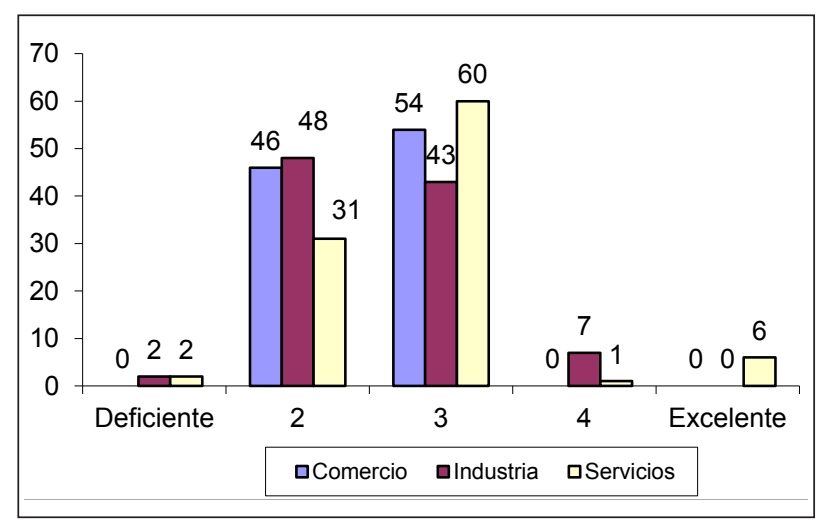

Fuente: Elaboración propia a partir de la EE.

Por último, y con el objetivo de obtener una calificación de la imagen que la opinión pública tiene de las empresas según el sector de actividad, se les solicitó a los encuestados que indicaran si las empresas de un sector eran más, menos o igual de socialmente responsable que las empresas de otro sector. Para su procesamiento, estas ramas fueron agrupadas según el porcentaje de respuestas afirmativas.

Cuadro 5. Calificación de la opinión pública según sector de actividad

\begin{tabular}{|l|l|}
\hline \multicolumn{1}{|c|}{ Imagen } & \multicolumn{1}{c|}{ Sector } \\
\hline $\begin{array}{l}\text { Alta } \\
\text { (obtuvieron más de 40\% } \\
\text { de respuestas afirmativas) }\end{array}$ & $\begin{array}{l}\text { Alimentos, Farmacéutica, } \\
\text { Salud y Telecomunica- } \\
\text { ciones }\end{array}$ \\
\hline $\begin{array}{l}\text { Media } \\
\text { (obtuvieron entre 29\% y } \\
\text { 39\% de respuestas afir- } \\
\text { mativas) }\end{array}$ & $\begin{array}{l}\text { Agricultura, Bebidas, Co- } \\
\text { mercio, Comunicaciones, } \\
\text { Energía, Química, Tecno- } \\
\text { logía y Transporte }\end{array}$ \\
\hline $\begin{array}{l}\text { Baja } \\
\text { (obtuvieron menos de 29\% } \\
\text { de respuestas afirmativas) }\end{array}$ & $\begin{array}{l}\text { Automotriz, Servicios fi- } \\
\text { nancieros y Vestimenta }\end{array}$ \\
\hline
\end{tabular}

Fuente: Elaboración propia a partir de la EE.

Así como la opinión pública identifica distintos niveles de RSE, las empresas presentan comportamientos diferenciados por su tamaño, sector de actividad y presencia de capitales extranjeros. 
El análisis preliminar de los datos de la encuesta aplicada a las empresas comprueba la existencia de esta diversidad (Ferre y otros, 2007). Las empresas del sector industrial se encuentran más involucradas en las prácticas de RSE, especialmente en aquellas vinculadas al medioambiente y al desarrollo formal de la organización. Se presenta la misma relación positiva entre RSE y las empresas de capital extranjero. El tamaño de las empresas muestra un comportamiento similar, aunque presenta mayor variabilidad, con resultados que alteran el orden "esperado" en muchas de las preguntas formuladas. Las empresas de capital nacional parecen tener un mayor grado de relacionamiento con asuntos sociales de interés comunitario.

\subsection{Percepción del rol de las empresas en la sociedad}

A fin de identificar los actores que la opinión pública y las empresas reconocen como responsables de distintas actividades beneficiosas para la sociedad, se consultó a los encuestados respecto a quién debería tener la mayor responsabilidad en "apoyar proyectos comunitarios y de caridad" y en "asegurar que la industria no dañe el medioambiente". Para el 71\% de la población en general, tanto el Gobierno como las grandes empresas son vistos como responsables de apoyar proyectos comunitarios y de caridad, mientras que para las empresas, este porcentaje disminuye en favor de una mayor responsabilidad del Gobierno. En cuanto a la tarea de asegurar

Gráfico 6. Opinión sobre los responsable de...

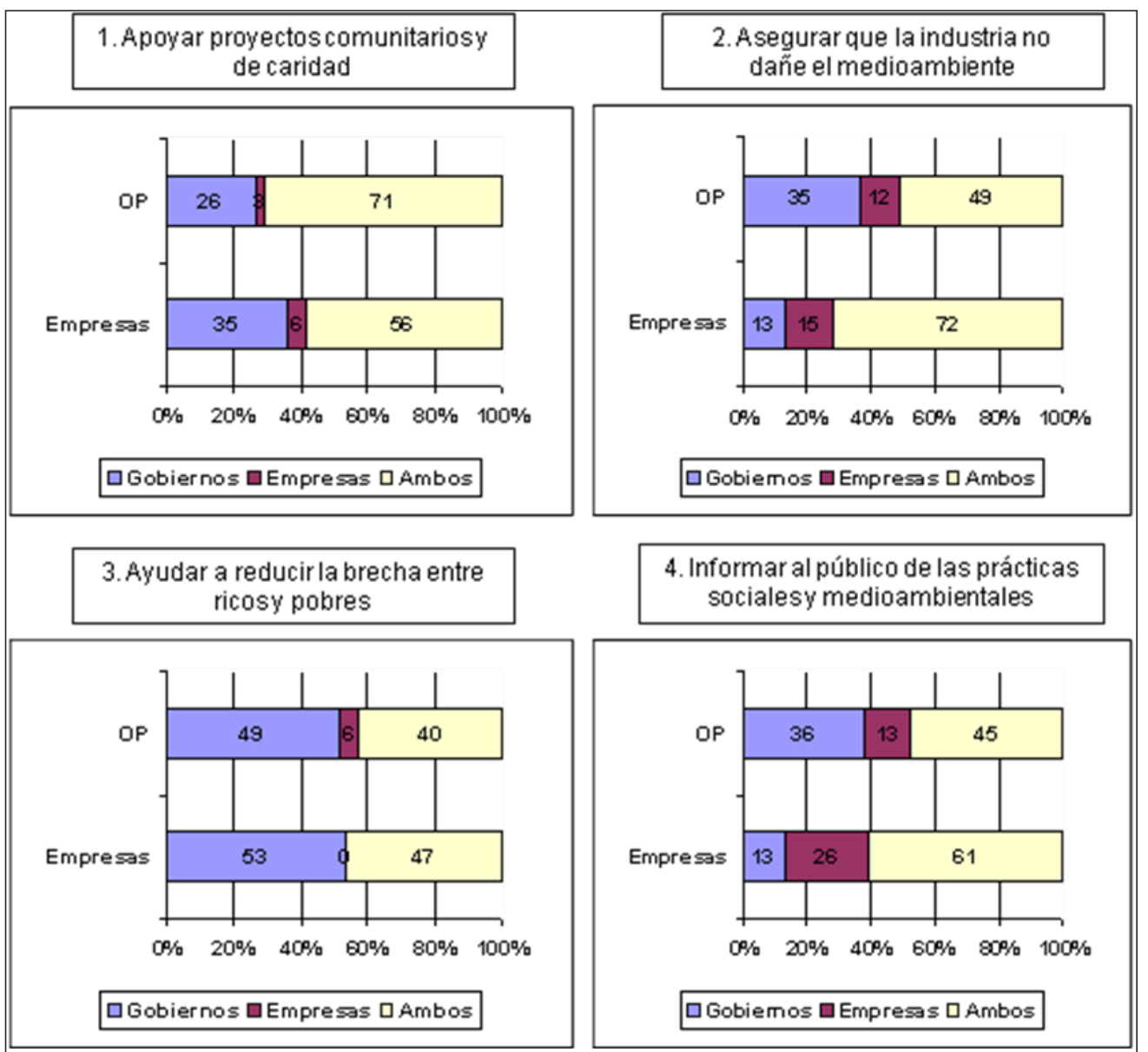

Fuente: Elaboración propia a partir de la EE. 
que la industria no dañe el medioambiente, más de un tercio de la población le asigna la principal responsabilidad al Gobierno, mientras que la opinión de las empresas las muestra más involucradas: $72 \%$ responde que la responsabilidad debería ser compartida entre las grandes empresas y el Gobierno.

Como era de esperar ante la pregunta: "ayudar a reducir la brecha entre ricos y pobres" tanto la opinión pública como las empresas le asignan mayor responsabilidad al gobierno y asignaron una calificación nula o casi nula a la responsabilidad asignada exclusivamente a las empresas. Por último, resulta interesante destacar la responsabilidad asignada a la acción de "informar al público de las prácticas sociales y medioambientales de las empresas". Mientras que la población le asigna un peso importante al gobierno como exclusivo responsable (36\%), las empresas destacan el papel que tienen en esta acción: 26\% opina que deben ser las empresas las que tiene la responsabilidad exclusiva en esta tarea y un $61 \%$ opina que la responsabilidad debe ser compartida entre gobierno y empresas. En la medida que las regulaciones de tipo ambiental generan costos para las empresas, este resultado podría indicar una cierta aversión a ese tipo de regulaciones.

\subsection{Dimensiones del concepto de responsabilidad social empresarial}

A fin de identificar la importancia relativa otorgada a las cuatro áreas en las que las empresas pueden actuar para mejorar su responsabilidad social ( -1 -clientes, proveedores y competidores, -2-accionistas, -3-empleados y -4-Estado, comunidad y medioambiente), se solicitó que los encuestados las ordenaran: ubicaron en primer lugar aquella que según su opinión debería ser el área más importante para la responsabilidad social, en segundo lugar el área que le sigue en importancia y así hasta llegar al área menos importante.

Resulta interesante notar que si bien la distribución de frecuencias es distinta, las áreas más valoradas para la opinión pública y empresas son: "Comunidad, Estado y Medioambiente" y los "Empleados". En tercer lugar los "Clientes, proveedores y competidores" y en último lugar los "Accionistas".

Gráfico 7. Área elegida como más importante para la RSE (\%)

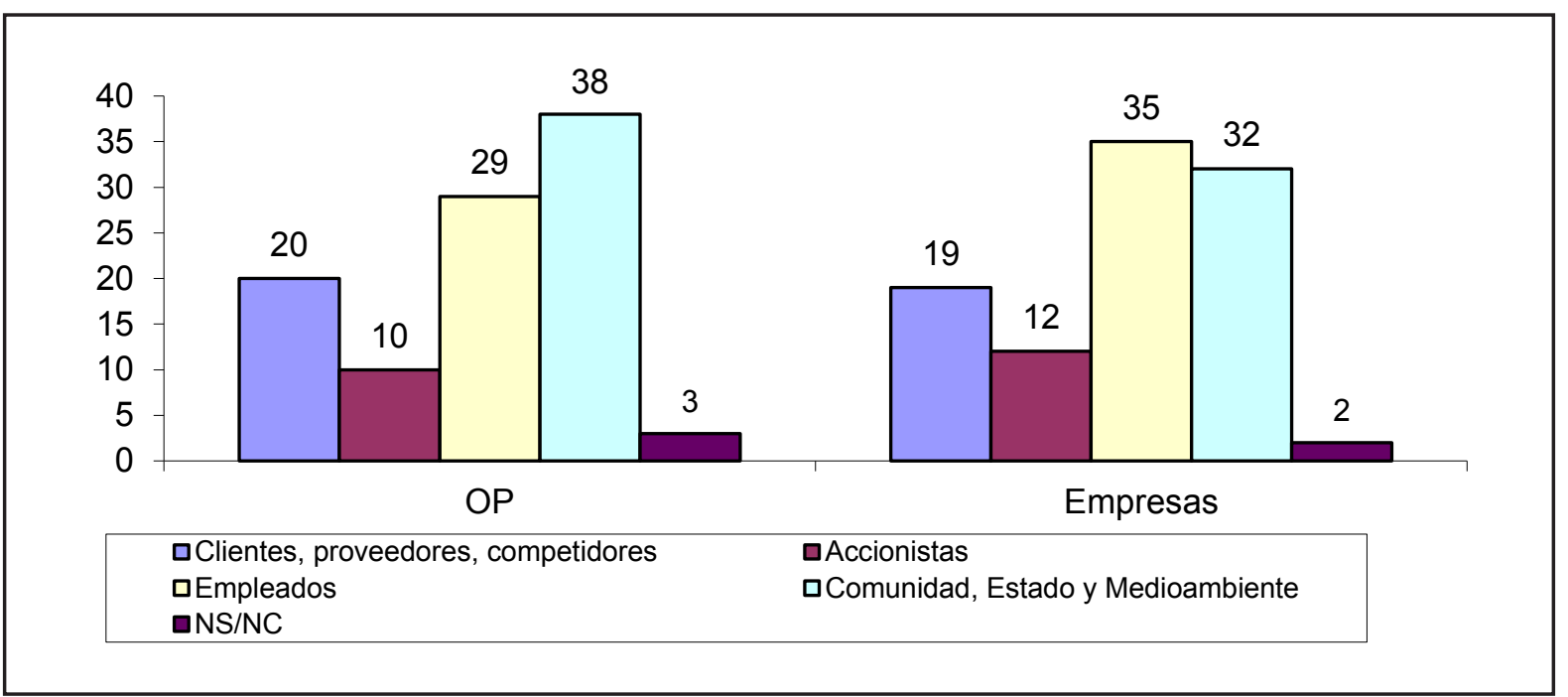

Fuente: Elaboración propia a partir de la EE 
Cuadro 6. Importancia que le otorga la opinión pública a las actividades de RSE que realizan las empresas uruguayas

\begin{tabular}{|c|c|c|}
\hline \multicolumn{2}{|c|}{ OP: Importancia dada a algunas actividades vinculadas a RSE } & $\begin{array}{c}\text { Muy } \\
\text { importantel } \\
\text { Importante (\%) }\end{array}$ \\
\hline \multirow{3}{*}{ Trabajadores / Empleados } & Bienestar de los trabajadores & 98 \\
\hline & Respeto a los derechos laborales & 97 \\
\hline & Tratar y pagar bien a sus empleados & 97 \\
\hline \multirow{3}{*}{ Comunidad / Estado / medioambiente } & Proteger el medioambiente & 96 \\
\hline & Realizar donaciones a causas sociales & 80 \\
\hline & $\begin{array}{l}\text { Contribuir a la comunidad, mas allá del cumpli }- \\
\text { miento de las leyes }\end{array}$ & 78 \\
\hline \multicolumn{2}{|c|}{ Empresas: Algunas actividades vinculadas a la RSE desarrolladas durante 2006} & $\begin{array}{l}\text { SI } \\
(\%)\end{array}$ \\
\hline \multirow{9}{*}{ Trabajadores / Empleados } & Capacitar y/o entrenar a los trabajadores & 85 \\
\hline & $\begin{array}{l}\text { Escuchar las sugerencias/ideas/opiniones de los } \\
\text { trabajadores }\end{array}$ & 69 \\
\hline & Mejorar el ambiente en el lugar de trabajo & 60 \\
\hline & Promover la participación e inclusión del trabajador & 56 \\
\hline & $\begin{array}{l}\text { Premiar a la productividad/rendimiento de los } \\
\text { trabajadores }\end{array}$ & 55 \\
\hline & $\begin{array}{l}\text { Mejorar la seguridad de los trabajadores en el lugar } \\
\text { de trabajo }\end{array}$ & 55 \\
\hline & Mejorar la salud de los trabajadores & 45 \\
\hline & Ayudar a las familias de los trabajadores & 33 \\
\hline & $\begin{array}{l}\text { Coparticipar a los trabajadores de los resultados } \\
\text { de la empresa }\end{array}$ & 15 \\
\hline \multirow{6}{*}{ Comunidad / Estado / medioambiente } & $\begin{array}{l}\text { El reciclaje o reutilización de materiales en los } \\
\text { procesos internos de producción }\end{array}$ & 59 \\
\hline & Mejorar las condiciones de vida de la comunidad & 52 \\
\hline & Apoyar a instituciones educativas & 48 \\
\hline & Apoyar programas sociales del Estado & 31 \\
\hline & Disminuir el impacto negativo en el medioambiente & 30 \\
\hline & Conjuntos con una ONG & 26 \\
\hline
\end{tabular}

Fuente: Elaboración propia a partir de la encuesta EOP y EE

La comparación de los resultados de ambas encuestas nos permite evaluar la correspondencia de las acciones empresariales en RSE con la valoración de la opinión pública sobre las áreas consideradas más importantes. Como se mencionó anteriormente, las acciones para mejorar las condiciones de los trabajadores y empleados son las que más se valorizan en la opinión pública. Coincidentemente, el 85\% de las empresas han realizado alguna instancia de capacitación y entrenamiento de sus empleados durante el año 2006. Entre un 55\% y un 70\% de las empresas han implementado acciones tendentes a promover la inclusión y el sentido 
de pertenencia de sus empleados (mejoras del ambiente de trabajo, escuchar opiniones, sugerencias, etc.). Aproximadamente entre un tercio y la mitad de las empresas, realizaron acciones tendientes a mejorar la salud de los trabajadores y sus familias y la seguridad en el lugar de trabajo. Este resultado refleja que en los temas de RSE el entorno más cercano es de suma importancia para la opinión pública, hecho que se ve reflejado en las acciones que las empresas priorizan.

\subsection{Decisión de compra y RSE}

Por último y con el objetivo de realizar un análisis preliminar de la influencia que tienen en la decisión de compra del público en general los aspectos vinculados a RSE, se presenta el siguiente cuadro.

Cuadro 7. Influencia en la decisión de compra de productos y servicios de ....

\begin{tabular}{|l|c|}
\hline \multicolumn{1}{|c|}{ OP } & $\begin{array}{c}\text { Extremada- } \\
\text { mente y muy } \\
\text { influyente (\%) }\end{array}$ \\
\hline $\begin{array}{l}\text {..la manera en que la empresa paga y } \\
\text { trata a sus empleados }\end{array}$ & 70 \\
\hline $\begin{array}{l}\text {..el impacto de los productos y/ o ser } \\
\text { vicios en la salud de los consumidores }\end{array}$ & 69 \\
\hline $\begin{array}{l}\text {..que la empresa sea reconocida por ser } \\
\text { socialmente responsable }\end{array}$ & 64 \\
\hline ..la nacionalidad u origen del producto & 61 \\
\hline $\begin{array}{l}\text {..el impacto de la empresa en el } \\
\text { medioambiente }\end{array}$ & 58 \\
\hline $\begin{array}{l}\text {..las contribuciones de la empresa a la } \\
\text { caridad y/ o causas sociales }\end{array}$ & 46 \\
\hline
\end{tabular}

Fuente: Elaboración propia a partir de la encuesta EOP

Como se observa en el cuadro, de acuerdo con la elaboración consiente del encuestado, todos los factores considerados influyen en la decisión de compra de los consumidores aunque con distinta intensidad. El análisis de las respuestas en las categorías más positivas ("extremadamente influyente" y "muy influyente") muestra, una vez más, el peso importante que tiene la relación de la empresa con sus empleados. Seguido del impacto de los productos y/o servicios en la "salud de los consumidores" (69\%) y en tercer lugar la influencia "que la empresa sea reconocida por ser socialmente responsable" (64\%). Resulta interesante, notar que menos de la mitad de los encuestados encontró como extremadamente influyente y muy influyente en su decisión de comprar, las contribuciones de la empresa a caridad y/o causas sociales.

\section{CONCLUSIONES}

En primer lugar, se destaca que se ha cumplido plenamente con el objetivo inicial de esta investigación, que proponía la descripción de la RSE en Uruguay basándose en datos objetivos y confiables que provinieran tanto desde la opinión pública como desde fuentes empresariales. En este sentido esta investigación constituye un claro aporte al tema, tanto en Uruguay como en la región.

Resulta interesante destacar que la mitad de la población declara haber escuchado hablar del tema de RSE. Como es esperable, este porcentaje aumenta en aquellos grupos más informados (más educados, población adulta, y ocupados).

La visión que las empresas tienen de sí mismas en cuanto a su desempeño en RSE es más crítica que la del público en general, donde la industria es el sector que evalúa más desfavorablemente dicho desempeño.

En lo que respecta a la percepción que se tiene del rol que deben cumplir las empresas y el gobierno en la sociedad, se encuentra que:

- Un alto porcentaje de ciudadanos observa que es una responsabilidad importante de las empresas y del gobierno el apoyar proyectos comunitarios y de caridad.

- En lo que respecta a los temas relacionados con el medioambiente, mientras que de la opinión pública surge que el gobierno tiene 
una responsabilidad muy alta en el tema, las empresas se asignan un rol importante. Esto podría indicar un cierto comportamiento estratégico por parte de las empresas, ya que la intervención del gobierno en regulaciones de tipo medioambientales les generaría costos adicionales. Esta situación podría llevar a que las empresas se interesen en mitigar dichos costos a través de un comportamiento amigable con el medioambiente.

Por último, y quizá el resultado que más se destaca de la comparación de ambas encuestas es la comprobación de la existencia de un cierto balance entre las acciones empresariales en RSE y la valoración que la opinión pública hace de cuáles son las áreas consideradas como las más importantes:

- La población otorga un valor importante a aquellos temas vinculados a su entorno más cercano: trabajadores y empleados. Este hecho se refleja en la importancia otorgada a los distintos aspectos que involucra la RSE, así como en la influencia que tiene la responsabilidad empresarial a la hora de decidir la compra de un producto o servicio.

- Se puede decir que las acciones priorizadas por las empresas se corresponden con el resultado anterior. Todas las acciones destinadas a capacitar, entrenar, promover la participación, mejorar la salud y la seguridad de los trabajadores alcanzan porcentajes mayores al 50\%.

\section{BIBLIOGRAFÍA}

Aparicio, J. y Valdés de la Vega, B. (2009). Sobre el concepto de responsabilidad social de las empresas. Un análisis europeo comparado. En: Cuadernos de Relaciones Laborales, Vol. 27, No 1, p. 53-75.

ACDE -Asociación Cristiana de Dirigentes de Empresas(2005). Índice Nacional de Responsabilidad Social Empresarial. [En línea] $3^{a}$ ed., Montevideo, 19p. Disponible en: http://www.acde.org.uy/comisiones\%20 de\%20trabajo/INFORME2005.doc. [Consultado marzo 10de 2010]
ACDE -Asociación Cristiana de Dirigentes de Empresas(2006). Índice Nacional de Responsabilidad Social Empresarial. [En línea] 4ª ed., Montevideo, 20p. Disponible en: http://www.acde.org.uy/comisiones\%20 de\%20trabajo/INFORME2006.doc. [Consultado marzo 10de 2010]

Bowen, Howard Rothmann (1953). Social Responsibilities of the Businessman. New York, Harper, 276 p.

Becchetti, L., Di Giacomo, S. y Pinnacchio, D. (2008). Corporate Social Responsibility and corporate performance: evidence from a panel of US listed companies. En: Applied Economics, Vol. 40, No 5, p. 541-567.

Carroll, A. (1998). Social Responsibility. En: Encyclopedic Dictionary of Business Ethics, Werhane and Freeman, Blackwell Publishers, Massachusetts, p. 593-595.

Commission of the European Communities (2001). Green paper: promoting a European framework for corporate social responsability. COM 366 final, junio, Bruselas, 32p.

De la Cuesta González, M. (2004). El porqué de la responsabilidad social corporativa. En: Boletín Económico de ICE, Secretaría de Estado de Comercio Exterior, Ministerio de Industria, Turismo y Comercio, España, No 2813, septiembre, p. 45-58.

Ferre, Z., Melgar, N., Pastori, H., Piani, G. y Rossi, M. (2007). La Responsabilidad Social Empresarial en Uruguay La visión desde las empresas, Documento de trabajo № 19, Departamento de Economía, FCSUdelaR, noviembre, 110p.

Fleishman-Hillard Inc. y NCL -US National Consumers League- (2005). Rethinking Corporate Social Responsibility, septiembre, 113p.

Institute of Social Ethical Accountability (2008). Responsabilidad Social 8000 (SA8000). [En línea] Nueva York, 12p. Disponible en: http://www.sa-intl.org/ data/n_0001/resources/live/2008StdSpanish.pdf. [Consultado el 05 de mayo de 2010].

Institute of Social Ethical Accountability (2008). AA1000 AccountAbility Principles Standard. [En línea] Disponible en: http://www.accountability.org/about-us/ publications/aa1000.html. [Consultado el 05 de mayo de 2010]. 
IUNT -Instituto Uruguayo de Normas Técnicas- (2010). Guía de Responsabilidad Social (ISO 26000:2010, IDT), 240p.

McWilliams, A., y Siegel, D. (2001). Responsabilidad Social Corporativa: una teoría desde la perspectiva de la empresa. En: Academy of Management Review, Vol. 26, No 1, p. $117-127$.

Piani, G., Ferre, Z., Rossi, M., Melgar, N. (2007). La Responsabilidad Social Empresarial en Uruguay. La visión desde la opinión pública. Documento de trabajo No 18, Departamento de Economía, FCSUdelaR noviembre, $142 \mathrm{p}$.

Porter, M. y Kramer, M. (2006). Strategy and Society: the link between competitive advantage and corporate social responsibility. En: Harvard Business Review, Vol. 84, No 12, p. 78-92.
PNUD - Prohumana (2002). Los chilenos opinan: Responsabilidad social de las empresas. Análisis de la Encuesta Mori sobre RSC, abril, Santiago de Chile, 63p.

United Nations Global Compact (1999). The ten principles. [En línea] United Nations Global Compact. Disponible en: http://www.unglobalcompact.org/ AbouttheGC/TheTENPrinciples/index.html. IConsultado el 05 de mayo de 2010].

Zadek, S. (2004). The path to Corporate Responsibility. En: Harvard Business Review, Vol. 82, No 12, p. 125-132. 